\title{
Field Discontinuity Refinement Criteria and Optimal Discretizations in Adaptive Finite-Element Electromagnetic Analysis for Microelectronic System Interconnections
}

\author{
Dennis Giannacopoulos
}

\begin{abstract}
The effectiveness of field-discontinuity refinement criteria for achieving optimal finite element discretizations is investigated. The criteria are first examined directly with finite-element solutions computed from optimally discretized systems. Subsequently, the optimality of the criteria are evaluated for practical adaptive finite-element electromagnetic analysis of principal device features in modern microelectronic system interconnection structures.
\end{abstract}

Index Terms-Adaptive systems, electromagnetic analysis, error analysis, finite-element methods.

\section{INTRODUCTION}

$\mathbf{I}$ $\mathrm{N}$ RECENT years, significant advances have been made on the electromagnetic modeling, simulation, and computer-aided design of microelectronic system interconnection (MSI) structures [1]. The main difficulty with computational MSI analysis is that a very large number of free-modeling parameters are needed to compute accurate and reliable simulations for realistic systems. The computational effort required for the electromagnetic analysis of the complex, dense, and irregularly routed arrays of high-speed interconnections that comprise modern MSI structures can often be prohibitive [1]. Yet such analyses are critical if MSI system performance is to be simulated with confidence. Currently, one promising way to overcome this computational barrier is by using adaptive solver technologies that are capable of intelligently evolving and improving an efficient distribution of DOF over the problem domain [2]. Moreover, the study of error distributions corresponding to optimal finite-element discretizations has recently attracted appreciable interest [2]-[5]. An incentive for this research focus stems from the potential benefits of identifying effective and reliable refinement criteria, for adaptive finite-element analysis (AFEA), based on a priori characterizations of optimal discretization solution properties. Such refinement criteria could be used in practice for efficiently guiding adaptive finite element electromagnetic solvers toward optimal accuracy solutions, without incurring the prohibitive computational costs associated with solving the optimal discretization

Manuscript received June 18, 2002. This work was supported by the Natural Sciences and Engineering Research Council of Canada.

The author is with the Department of Electrical and Computer Engineering, McGill University, Montreal, PQ H3A 2A7, Canada (e-mail: dennis.giannacopoulos@mcgill.ca).

Digital Object Identifier 10.1109/TMAG.2003.810383 problem directly [2]. This may be useful for overcoming the current MSI computational bottleneck.

\section{FiELD DiscontinUity IN Optimal DiscretizATIONS}

The primary purpose of the present contribution is to investigate the ability of practical field-discontinuity refinement criteria to effectively reproduce the main modeling characteristics of optimal finite-element discretizations. While field-discontinuity refinement criteria are widely used and have been shown to be among the most effective for AFEA in electromagnetics, their exact connection to optimal finite-element discretizations has yet to be reported in the mainstream literature.

In this paper, it is shown directly for the first time that the field-discontinuity criteria considered can correctly identify optimal error distributions, without the expense of solving the optimal discretization problem. Specifically, it is demonstrated that field discontinuity is satisfied precisely for optimally discretized problem domains, whereas it is not for nonoptimal meshes. It should be noted that the experimental data supporting this conclusion are based on field solutions obtained using an explicit formulation for computing optimal discretizations directly; thus, the results computed under these conditions cannot be refuted. Namely, the formulation derived for general scalar Helmholtz systems in [3] was employed, which is based on simultaneously satisfying sets of optimization equations defined for both the geometric discretization parameters (i.e., element vertex positions) and the field solution unknowns. In contrast, previous adaption studies reported with practical, but nonoptimal discretizations, have not been able to provide the sufficiently rigorous conditions required for examining the relationship between field discontinuity refinement criteria and optimal finite-element discretizations. Therefore, the novelty of this work is, in part, the use of optimally discretized benchmark systems for evaluating this relationship, a critical component in obtaining the definitive data required.

In addition, the operational value of field-discontinuity refinement criteria is evaluated for practical electromagnetic AFEA of principal device features present in modern MSI structures, which are known to pose challenging problems in numerical modeling. Although these types of refinement criteria are well established, the study of their effectiveness in AFEA for electromagnetic simulation of MSI is still relatively new and can provide valuable insight. 
TABLE I

NUMERICAL RESULTS FOR POINT SINGULARITY BENCHMARK

\begin{tabular}{ccccc}
\hline & \multicolumn{2}{c}{ Uniform Meshes } & \multicolumn{2}{c}{ Optimal Meshes } \\
\cline { 2 - 5 } No. Elements & $F$ Error & $E$ Error & $F$ Error & $E$ Error \\
\hline & & & & \\
2 & $540.6 \%$ & 0.6470 & $35.87 \%$ & 0.0000 \\
4 & $242.5 \%$ & 0.4160 & $3.756 \%$ & 0.0000 \\
6 & $147.9 \%$ & 0.3605 & $0.8521 \%$ & 0.0000 \\
8 & $102.1 \%$ & 0.3313 & $0.2840 \%$ & 0.0000 \\
10 & $75.34 \%$ & 0.3108 & $0.1192 \%$ & 0.0000 \\
12 & $58.01 \%$ & 0.2994 & $0.0583 \%$ & 0.0000 \\
14 & $46.01 \%$ & 0.2801 & $0.0317 \%$ & 0.0000 \\
16 & $37.29 \%$ & 0.2673 & $0.0187 \%$ & 0.0000 \\
\hline
\end{tabular}

\section{RESULTS}

Two simple one-dimensional (1-D) free-space examples are presented first, in order to examine the effectiveness of field-discontinuity measures for identifying optimal finite element error distributions. Subsequently, the performance of field-discontinuity refinement criteria is compared to that of optimal discretization-based refinement criteria for practical electromagnetic AFEA using a two-dimensional (2-D) Laplace test system. Finally, a three-dimensional (3-D) high-frequency structure is considered in order to confirm the potential implications of AFEA based on field-discontinuity refinement criteria for the electromagnetic simulation of MSI structures.

\section{A. 1-D: Free-Space Singularity Test Systems}

These static benchmark systems were first studied in [6] and are based on the classical point and line singularity models in free space. The objective for each benchmark system is to compute the functional value based on the resolution of a radial neighborhood close to the singularity and spanning a 100-fold decay in potential: the point charge and line current, of magnitudes $10^{-9} / 9 \mathrm{C}$ and $5 \times 10^{6} \mathrm{~A}$, respectively, are located at the origin, and the two boundaries of both problem domains are set at radial distances of 0.1 and $10 \mathrm{~m}$ away from the singular source distributions. It may be noted that the field solutions associated with the free-space point charge and line current models contain the types of singularities associated with the sharp material corners and edges that are present in many practical MSI structures [2]. Thus, the primary feature of these test systems is the rapid field solution variation close to the singularity, which has been shown to drastically reduce the finite-element convergence rate.

The results for the point and line singularity models are given in Tables I and II, respectively. To focus ideas, a field discontinuity error measure [7] was evaluated for both uniform and globally optimal second-order meshes. All results were computed using standard second-order Lagrangian elements for the analysis of these 1-D examples. Furthermore, the results corresponding to the optimal meshes are based on solving the optimization equations for the geometric discretization parameters [3], as previously explained. Hence, each of the optimal discretization results computed for these two benchmark systems represents the ideal mesh for a given number of DOF, i.e., the mesh that produces the most accurate solution possible for the
TABLE II

NUMERICAL RESULTS FOR LINE SINGULARITY BENCHMARK

\begin{tabular}{ccccc}
\hline & \multicolumn{2}{c}{ Uniform Meshes } & \multicolumn{2}{c}{ Optimal Meshes } \\
\cline { 2 - 5 } No. Elements & $F$ Error & $H$ Error & $F$ Error & $H$ Error \\
\hline & & & & \\
2 & $33.09 \%$ & 0.2148 & $9.315 \%$ & 0.0000 \\
4 & $16.73 \%$ & 0.1139 & $0.8409 \%$ & 0.0000 \\
6 & $10.44 \%$ & 0.0859 & $0.1801 \%$ & 0.0000 \\
8 & $7.155 \%$ & 0.0706 & $0.0587 \%$ & 0.0000 \\
10 & $5.175 \%$ & 0.0602 & $0.0244 \%$ & 0.0000 \\
12 & $3.883 \%$ & 0.0525 & $0.0118 \%$ & 0.0000 \\
14 & $2.994 \%$ & 0.0463 & $0.0064 \%$ & 0.0000 \\
16 & $2.357 \%$ & 0.0413 & $0.0038 \%$ & 0.0000 \\
\hline
\end{tabular}

variational finite element formulation used [3]. In each case, $F$ Error indicates the error in the global functional value. For the results reported in Table I, $E$ Error indicates the average error in the electric field continuity at the element interfaces. Similarly, in Table II, $H$ Error indicates the average error in the magnetic field continuity at the element interfaces. It should be noted that for the uniform discretizations, the nonzero field-discontinuity quantities correctly indicate a large error for the functional value. However, for the optimal discretizations, the field-discontinuity errors are identically zero. Hence, field discontinuity is satisfied exactly with respect to these optimal finite element solutions. For each of the uniform and optimal second-order solutions considered, the average error in field discontinuity was calculated as the mean of the difference in field values over each individual element interface. Hence, the field discontinuity criteria evaluated for these 1-D test systems confirm that they can correctly identify optimal error distributions for electrostatic and magnetostatic finite element solutions. Although the principle of using field discontinuity for characterizing optimal finite element discretizations appears to be quite effective for the benchmark systems considered, it would not be prudent to recommend this principle for general use without providing theoretical justification or further investigating its effectiveness for a wider range of practical problems. Therefore, the practical value of this approach is considered next.

\section{B. 2-D Laplace Test System}

A 2-D test system is examined to investigate the practical value of using field-discontinuity refinement criteria to identify optimal finite element discretizations. Specifically, the performance of field-discontinuity refinement criteria is compared to that of optimal discretization-based refinement criteria for practical electromagnetic AFEA using a 2-D Laplace test system. The Laplace benchmark system is described by Fig. 1. It is one-quarter of a square coaxial line in cross section-the standard "L" problem. The conductor boundary conditions are 1 and $0 \mathrm{~V}$ as indicated, and the symmetry planes are labeled $N$.

Performance results for first-order $h$-adaption studies are presented in Fig. 2. In addition to the uniform $h$-refinement baseline (initial mesh uniformly subdivided to add elements to the discretization), a practical field discontinuity-based $h$-adaption [7] and an optimal discretization based $h$-adaption (Type-A [3]) result are included for comparison. Specifically, 


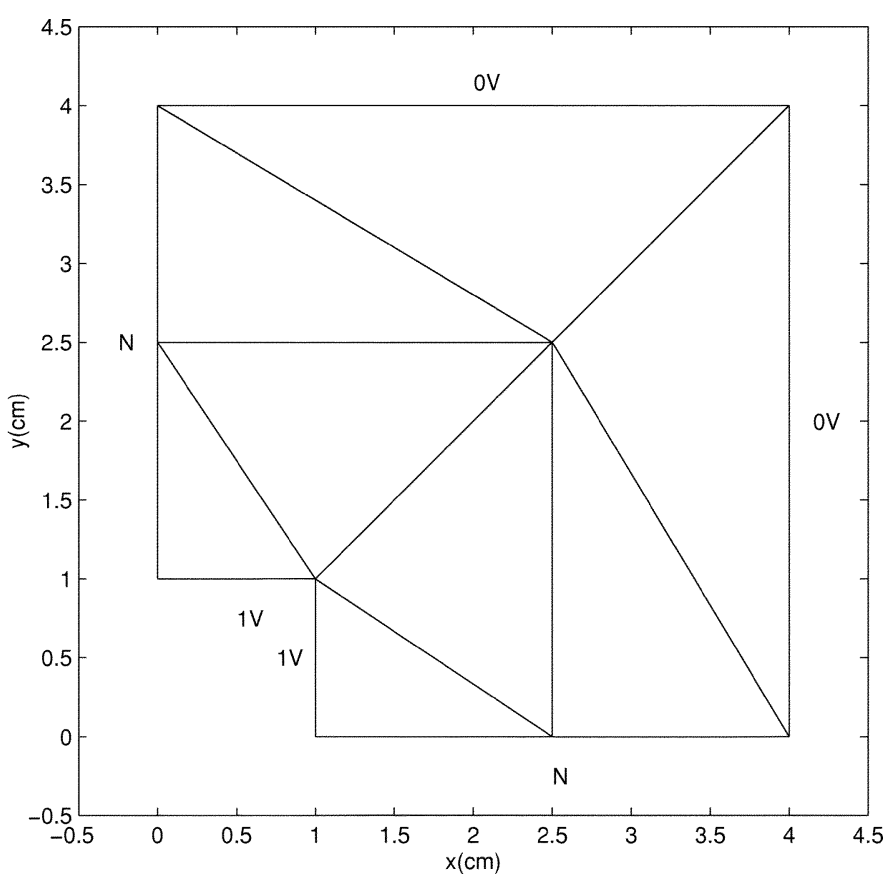

Fig. 1. Laplace benchmark system: Initial $h$ mesh (eight triangles).

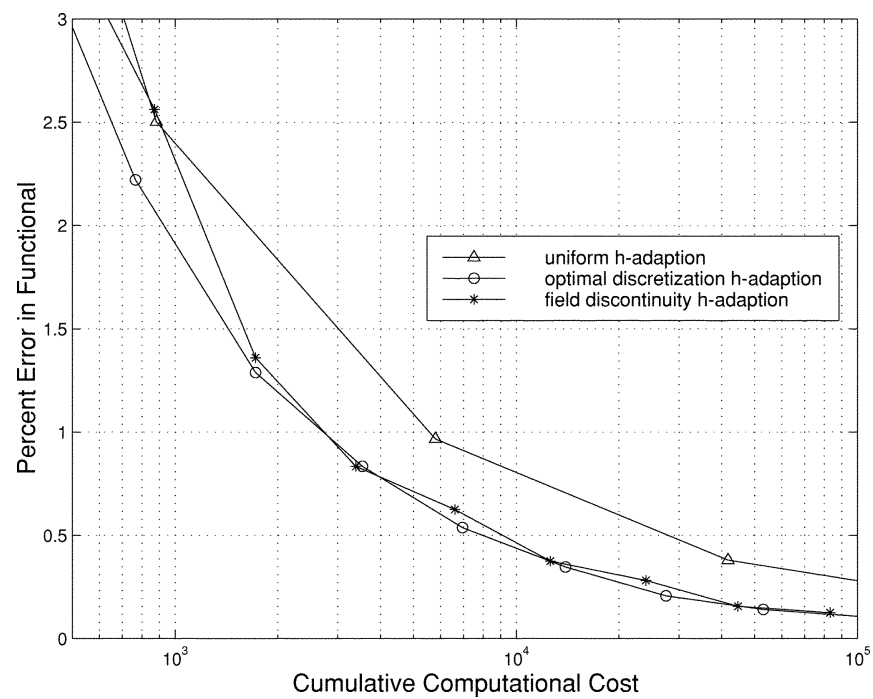

Fig. 2. Cumulative cost of adaption versus percent error in functional for the Laplace system.

for the latter the relative discretization errors over a nonoptimal finite element mesh can be estimated in terms of how well the optimization equations corresponding to the geometric discretization parameters are satisfied by that discretization. These optimization equations are defined elementwise, and they can be evaluated locally to indicate the relative optimality of the elements in a mesh. Hence, the optimal discretization-based refinement criteria are defined implicitly as measures of the residuals of the geometric optimization equations, which will evaluate identically to zero for an optimal mesh. Note: all the results shown in Fig. 2 correspond to the initial mesh of Fig. 1. A 50\% increment in the number of DOF per adaptive step was used for these $h$-adaption results. An example $h$-adapted mesh is shown in Fig. 3 for reference.

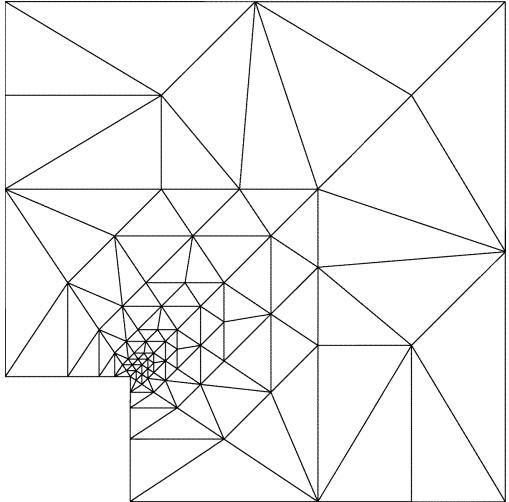

Fig. 3. Example refinement due to $h$-adaption for Laplace system.

It should be noted that the optimal discretization-based refinement criteria that were used for comparison purposes are directly related to the optimality of a finite element discretization, and they have been shown to be effective in guiding practical AFEA procedures toward optimal discretizations [3]. Furthermore, the performance results for the field discontinuity-based refinement criteria shown in Fig. 2 are comparable to those of the optimal discretization based criteria. Therefore, these findings corroborate the 1-D results presented above that field discontinuity-based refinement criteria can correctly identify optimal finite-element discretizations and, further, that they may be used to adaptively evolve the main modeling characteristics of optimal finite element discretizations.

\section{3-D: Microstrip Filter Structure}

A 3-D test system is considered in order to confirm the potential value of field discontinuity refinement criteria in AFEA for the electromagnetic simulation of MSI structures. The system is a microstrip low-pass filter of the type that commonly occurs in monolithic integrated circuits [1]. This single-layer structure consists of three rectangular microstrips printed on a dielectric substrate. The system is fully shielded, backed by a ground plane from below, and terminated $3 \mathrm{~mm}$ above the substrate. The dielectric has a thickness of $1 \mathrm{~mm}$ and a relative permittivity of 2.2. The layout of the strips is described by Fig. 4 . The surface of the substrate is 20 by $20 \mathrm{~mm}$, and the strips are $2 \mathrm{~mm}$ wide. The port strips are $9 \mathrm{~mm}$ long with their centers offset $6 \mathrm{~mm}$ from the sides of the structure, and the central strip is $16 \mathrm{~mm}$ long. The primary features of this test system are the sharp corners that occur at the strip terminations and at the strip-to-strip junctions, which are typical in MSI structures and can introduce mathematical singularities in the FEM field models. The objective of the analysis is to resolve the magnitude of $S_{12}$ at a prescribed operating frequency.

This MSI structure requires a 3-D high-frequency solution and is well suited to full-wave vector field analysis. All computations reported in this section were obtained using first-order edge-element tetrahedra to solve for the electric field. Results are reported for $h$-adaption studies based upon a standard field-discontinuity error estimator [7]. The $h$-adaption results are based on refining $50 \%$ of the tetrahedra in the mesh at each adaptive step, and the initial mesh is shown in Fig. 4. The convergence of the error in the magnitude of $S_{12}($ at $7.44 \mathrm{GHz})$ 


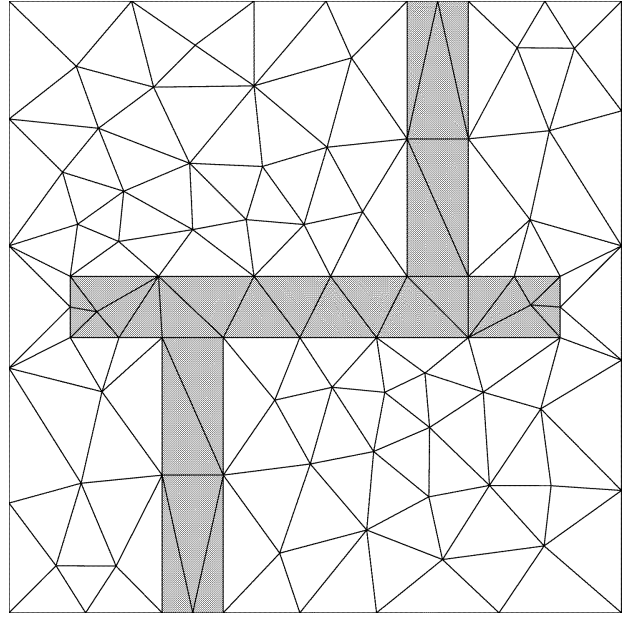

Fig. 4. The initial tetrahedral mesh used for the $h$-adaption studies for the microstrip filter structure is illustrated with a sectional view of the element faces that lie in the plane of the strips (shaded), on the substrate surface. The mesh consists of 1017 tetrahedra.

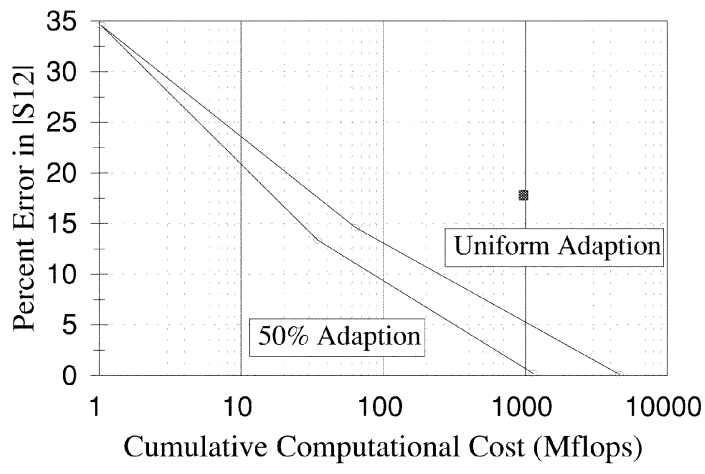

Fig. 5. The convergence of the error in the magnitude of $S_{12}$ versus computational cost for $h$-adaption studies of the microstrip filter structure are plotted. Both uniform adaption and 50\% update adaption (field discontinuity error indicator) results are provided. The single square knot represents the result for the nonadaptive mesh described by the right panel in Fig. 6. Note: All costs are for a complex profile Gaussian elimination solver.

versus computational cost is plotted in Fig. 5, to illustrate the potential advantages of directed $h$ adaption over uniform refinements. For example, $1 \%$ error results can be computed using 50\% $h$ adaption for less than one-quarter of the cost of uniform adaption. A sample $h$-adaption mesh from this study is described in Fig. 6 (left panel). Also, the single square knot result in Fig. 5 corresponds to the nonadaptive intuitively reasonable strip-focused discretization shown in Fig. 6 (right panel) and is included for comparison. Finally, it is important to note that the intuitively reasonable nonuniform mesh result is significantly inferior to both the field-discontinuity driven $h$ and the uniform adaption results.

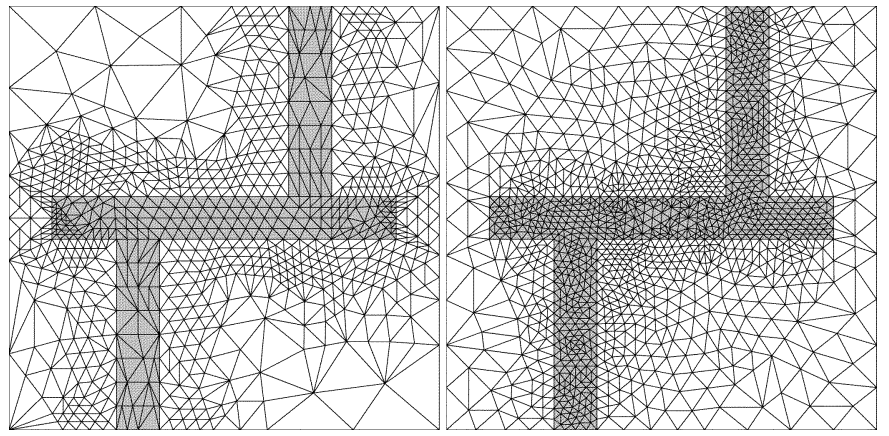

Fig. 6. An example of an $h$-adaption mesh (left) and the nonadaptive nonuniform strip-focused mesh (right), produced in the study reported by Fig. 5, are shown with a sectional view of the element faces that lie in the plane of the strips (shaded) on the substrate surface. The $h$-adaption mesh (left) was generated from the initial mesh described by Fig. 4, using a field discontinuity error indicator, with standard 8:1 element subdivisions and follow-up Delaunay mesh corrections; it consists of 30948 tetrahedra. The nonadaptive mesh (right) consists of 31004 tetrahedra.

\section{CONCLUSION}

In this work, previously established field discontinuity refinement criteria were shown to directly correspond to optimal error distributions for finite element electromagnetic solutions. Moreover, the performance of these types of refinement criteria compared to refinement criteria derived expressly from the optimal discretization principle itself was examined for practical AFEA of the electromagnetic behavior of key MSI device features. The the field discontinuity refinement criteria were shown to be effective in guiding practical AFEA procedures toward optimal finite-element discretizations. In addition, the potential value of using field discontinuity refinement criteria for the 3-D electromagnetic AFEA of practical high-frequency MSI structures was illustrated.

\section{REFERENCES}

[1] A. Polycarpou, P. Tirkas, and C. Balanis, "The finite-element method for modeling circuits \& interconnects for electronic packaging," IEEE Trans. Microwave Theory Tech., vol. 45, pp. 1868-1874, Oct. 1997.

[2] D. Giannacopoulos, "Toward optimal error distributions in adaptive finite element electromagnetic analysis for microelectronic interconnection structures," IEEE Trans. Magn., vol. 38, pp. 401-404, Mar. 2002.

[3] — - "Optimal discretization-based adaptive finite element analysis for electromagnetics," Ph.D. dissertation, McGill Univ., Montreal, Canada, 1998.

[4] L. Janicke, A. Kost, and P. A. Bastos, "Local error distribution in adaptively generated meshes," IEEE Trans. Magn., vol. 36, pp. 1627-1630, July 2000.

[5] L. Y. Li and P. Bettess, "Notes on mesh optimal criteria in adaptive finite element computations," Com. Num. Meth. Eng., vol. 11, pp. 911-915, 1995.

[6] D. Giannacopoulos and S. McFee, "Toward optimal $h-p$ adaption near singularities in finite element electromagnetics," IEEE Trans. Magn., vol. 30, pp. 3523-3526, Sept. 1994.

[7] J. P. Webb and B. Forghani, "Adaptive improvement of magnetic fields using hierarchal tetrahedral finite elements," IEEE Trans. Magn., vol. 30, pp. 3511-3514, Sept. 1994. 